\title{
El comportamiento bioproductivo de cerdas reproductoras y su descendencia alimentadas con aditivo probiótico
}

\author{
The bioproductive behavior of breeding sows and their offspring fed with probiotic additive
}

\author{
José Efraín Miranda-Yuquilema; ; Alfredo Marin-Cárdenas²; Mabel González-Pérez ${ }^{3}$
}

1 M.Sc. Becario del Instituto de Fomento a Talento Humano, SENESCYT, Universidad Central "Marta Abreu” de Las Villas, Santa Clara, Cuba, efra_miranda@outlook.com.

2 Ph.D. Universidad Central "Marta Abreu” de Las Villas, Santa Clara, Cuba, alfredomc@uclv.edu.cu.

3 Ingeniero Pecuario. Centro de Investigaciones Agropecuarias. Universidad Central "Marta Abreu” de Las Villas. Santa Clara, Cuba. mabelgp@uclv.edu.cu.

Citar: Miranda-Yuquilema, J.E., Marin-Cárdenas, A. \& González-Pérez, M. (2018). El comportamiento bioproductivo de cerdas reproductoras y su descendencia alimentadas con aditivo probiótico. Rev. Cienc. Agr. 35(1): 69-81. doi: http://dx.doi.org/10.22267/rcia.183501.84.

Recibido: Marzo 18 de $2017 . \quad$ Aceptado: Noviembre 20 de 2017.

\section{RESUMEN}

Los aditivos probióticos contienen microorganismos benéficos vivos que incluidos en la dieta en cantidades adecuadas repercuten de manera positiva en la salud del huésped. Sin embargo, los altos costos han limitado su uso en los productores de carne de cerdo en los países en desarrollo. Con el objetivo de evaluar el comportamiento bioproductivo en cerdas reproductoras y su descendencia al suplementar biopreparado en la dieta. Se emplearon 12 cerdas reproductoras híbridos CC21 (Yorkshire-Landrace/L35 Duroc) y su descendencia, distribuidas en dos grupos de síes animales cada uno, T1 Control y T2 tratamiento. T2, contenía Lactobacillus acidophilus, Streptococcus thermophilus y Kluyveromyces fragilis (L-4 UCLV). Se utilizó un Diseño Completamente Aleatorizado, donde se evaluó la ganancia de peso y el comportamiento productivo en la descendencia; ganancia de peso en las crías a partir del nacimiento hasta la pre-ceba, casos diarreicos y mortalidad, y la caracterización morfotintorial de los microrganismos provenientes del hisopado rectal. La ganancia de peso en cerdas madres y su descendencia fue mayor $(\mathrm{P}>0,05)$ en el $\mathrm{T} 2$. Los casos diarreicos y mortalidad fue menor $(\mathrm{p}<0,05)$ en el T2; con la caracterización morfo-tintorial se observó mayor $(\mathrm{P}<0,05)$ carga microbiana principalmente de cocos en cadena y aislados, bacilos cortos y en cadena Gram positivo y levaduras. Se concluye, que la inclusión de biopreparado en la dieta de cerdas reproductorasy su descendencia mejora el comportamiento bioproductivo, reduce los trastornos diarreicos y muertes, y aumenta la carga microbiana con característica morfo-tintorial correspondientes a bacilos, cocos Gram positivo y levaduras.

Palabras clave: Bacterias ácido-lácticas, característica morfo-tintorial, ganancia de peso, levaduras, salud. 


\begin{abstract}
Probiotic additives contain beneficial microorganisms that included in the diet, in sufficient quantities, have a positive effect on the health of the host. However, high costs have limited its use in pork producers in developing countries. With the objective of evaluating the bioproductive behavior in breeding sows and their offspring when bioprepared in the diet is complemented. We used 12 hybrid breeding sows CC21 (Yorkshire-Landrace/L35 Duroc) and their descendants, divided into two groups of six animals each. T1, control and T2, treatment. T2, contained Lactobacillus acidophilus, Streptococcus thermophilus and Kluyveromyces fragilis (L-4 UCLV). A completely randomized design was used, the weight gained and the reproductive behavior in the offspring were evaluated; Weight gain in piglets from birth to pre-fattening, diarrheal cases and mortality, and the morpho-teintorial characterization of the microorganisms from the rectal swab. The weight gain in mother sows and piglets was higher $(\mathrm{P}>0.05)$ in $\mathrm{T} 2$. The diarrheic cases and mortality were lower $(\mathrm{P}<0.05)$ in $\mathrm{T} 2$; with the morpho-teintorial characterization, higher microbial load $(\mathrm{P}<0.05)$ was observed mainly coconuts in chain and isolated, short and chain bacilli positive gram and yeasts. It is concluded that the inclusion of bioprepared in the diet of the breeding sows and their offspring improves the bioproductive behavior, reduces diarrhea and deaths and increases the microbial load with corresponding morpho-teintorial characteristic to Bacilli, gram-positive cocci and yeasts.
\end{abstract}

Key words: Lactic acid-bacteria, morpho-teintorial characteristic, weight gain, yeasts, health.

\section{INTRODUCCIÓN}

En la última década la industria porcina se ha preocupado por la eficiencia productiva, aumentando el número de crías por hembra al año a un costo menor, lo cual implica el acortamiento de los días de lactancia, así como el tiempo del destete en plena etapa de desarrollo fisiológico de los lechones (Yin et al., 2014). Dicha situación conlleva al tardío establecimiento de la microbiota natural en el tracto digestivo, que provoca la consecuente aparición de enfermedades entéricas, debido a la rápida colonización de microorganismos patógenos en dichos animales (Chaytor et al., 2011; Ihara et al., 2013).

En las décadas pasadas, el método de mayor uso para prevenir enfermedades entéricas fue el uso de antibióticos como aditivos alimentarios. Varios estudios han comprobado las influencias negativas de éstos en la eubiosis del sistema gastrointestinal, dando lugar a la resistencia bacteriana a fármacos y su presencia residual en las carnes, leche, huevos y otros productos de origen animal, afectando así la salud humana al consumirlos, de ahí que sea necesario introducir los probióticos como una alternativa ante esta problemática (García et al., 2012).

La inclusión de los probióticos en la dieta de los animales, podría favorecer a mejorar los rendimientos productivos, así como de la salud del huésped (Miranda et al., 2017). Una de las alternativas de los probióticos es ejercer su efecto, principalmente en la exclusión de microorganismos patógenos, gracias a su efecto antimicrobiano y la producción de ácido láctico, creando un ambiente favorable para la respuesta inmunológica y la prevención de enfermedades infecciosas en el hospedero (Cottney et al., 2012). Diversos países europeos y de América Latina, estudian los efectos de los microorganismos eficientes en algunas especies de animales, como ganado bovino, porcino, ovino y aves, puesto que han logrado mejorar los índices bioproductivos (Sun et al., 2015). El objetivo del presente estudio fue evaluar el comportamiento bioproductivo de cerdas reproductoras y su descendencia al incluir probiótico en la dieta. 


\section{MATERIALES Y MÉTODOS}

Área de estudio. La obtención del preparado microbiano y las pruebas microbiológicas se realizó en el Laboratorio de Fermentaciones, Centro de Investigaciones Agropecuarias (CIAP) y Laboratorio de Microbiología, Facultad de Ciencias Agropecuarias, Universidad Central "Marta Abreu" de Las Villas (Cuba).

El estudio de campo se desarrolló en la unidad de producción porcina "Javierito", poblado de Mataguá, Villa Clara (Cuba), localizado a $22^{\circ} 09^{\prime} 00^{\prime \prime} \mathrm{LN}$ $79^{\circ} 58^{\prime} 35^{\prime \prime} \mathrm{LO}, 230 \mathrm{msnm}$, con una precipitación anual de $115 \mathrm{~mm}$, temperatura media de $24,2^{\circ} \mathrm{C}$ y humedad relativa anual de $82,97 \%$.

Diseño y tratamientos experimentales. Se utilizó un Diseño Completamente Aleatorizado con síes repeticiones por tratamiento, donde cada animal representó una unidad experimental. Los tratamientos evaluados fueron: Control, dieta basal sin aditivo (T1) y Tratados, dieta basal más aditivo microbiano (T2).
Animales y dieta basal. Se emplearon 12 cerdas reproductoras híbridos CC21 (Yorkshire - Landrace/ L35 Duroc) con peso vivo $165 \pm 3 \mathrm{~kg}$, todas del tercer parto. Con seis animales por tratamiento y tras el parto, los cerditos se distribuyeron al azar para conformar los grupos experimentales con 60 animales por tratamiento (30 hembras y 30 machos). Las cerdas madre a partir de 110 días de gestación hasta el destete, se ubicaron en corrales independiente (maternidad). Los lechones a partir del destete se reagruparon (sin alterar los grupos de procedencia) con 20 cerditos en corrales de $6 \times 6,5 \mathrm{~m}$ pisos de cemento. El alimento balanceado empleado se puede apreciar en la Tabla 1, que cumple con los requerimientos nutricionales para cerdos según la categoría animal (NRC, 1998). Este se ofreció a las reproductoras dos veces por día, a las 7:00 am y 4:00 pm. y ad libitum a los cerditos a partir de los siete días de edad hasta el destete y de ahí hasta 70 días de edad, se alimentaron en el mismo horario que a sus madres. El agua, también, se suministró ad libitum en bebederos tipo tetinas.

Tabla 1. Composición bromatológica del alimento ofrecido a los animales en estudio.

\begin{tabular}{lccccc}
\hline \multicolumn{1}{c}{ Indicadores } & $\begin{array}{c}\text { \% Proteína } \\
\text { cruda }\end{array}$ & $\begin{array}{c}\text { \% Extracto } \\
\text { etéreo }\end{array}$ & $\begin{array}{c}\text { \% Fibra } \\
\text { cruda }\end{array}$ & $\begin{array}{c}\text { \% Materia } \\
\text { orgánica }\end{array}$ & $\begin{array}{c}\text { \% Materia } \\
\text { seca }\end{array}$ \\
\hline Neonato & 20 & 5 & 4 & 7 & 90 \\
Destete & 19 & 4 & 3 & 6 & 90 \\
Preceba & 17 & 3,8 & 3,6 & 4 & 90 \\
Cerdas gestantes & 12 & 3,2 & 6,4 & 6,1 & 87 \\
Cerdas lactantes & 16,8 & 5,3 & 5,2 & 7,1 & 90 \\
\hline
\end{tabular}


Sistema de manejo de los animales. La maternidad se mantuvo a $28^{\circ} \mathrm{C}$ de temperatura durante las primeras dos semanas después de parto, posteriormente se redujo en $1,5^{\circ} \mathrm{C}$ cada semana hasta el destete. El fotoperiodo fue controlado con $12 \mathrm{~h}$ de luz y $12 \mathrm{~h}$ de oscuridad. Las camadas de cada tratamiento se ubicaron distantes unas de otras (con un cuartón intermedio a ambos lados del pasillo) para evitar la auto inoculación. Los lechones se destetaron a los 33 días de edad. Los cerditos provenientes de cada grupo en estudio recibieron las atenciones veterinarias pertinentes según el Manual de Manejo de Hembras y Primerizas (Coates et al., 2013).

Preparados microbianos. Las cepas utilizadas para la obtención del preparado microbiano fueron: Lactobacillus acidophilus, Estreptococcus thermophilus, fueron provenientes del banco de cepas Villa Clara y Kluyveromyces fragilis (L-4 UCLV) banco de cepas de la Universidad Central "Marta Abreu" de Las Villas, Santa Clara (Cuba). Estas se activaron en leche descremada a $37^{\circ} \mathrm{C}$ por $24 \mathrm{~h}$. Para la obtención del preparado microbiano, se utilizaron los siguientes sustratos: melaza de caña de azúcar más yogurt de soya y levadura de torula y esto se fermentó a $37^{\circ} \mathrm{C}$ por $24 \mathrm{~h}$, según la técnica descrita por Miranda et al., (2017). La composición química y la concentración microbiana del preparado microbiano empleado para los animales, fue la siguiente: $13 \%$ materia seca, $16 \%$ proteína gruda, $13,5 \%$ proteína verdadera, 3,1\% extracto etéreo,

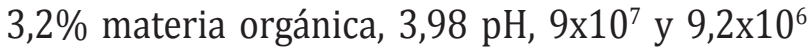
(bacterias y levaduras) UFC/mL de concentración microbiana, 0,73 $\mathrm{mmol} / \mathrm{mL}$ de ácido láctico y $94 \%$ de viabilidad.

\section{Suministro de aditivo microbiano a los anima-}

les. El aditivo microbiano, se aplicó a las 7:00 am en intervalo de tres días. Las cerdas reproductoras del grupo $\mathrm{T} 2$ recibieron $20 \mathrm{~mL}$ de preparado microbiano, inoculado en 0,5kg de dieta basal y 0,6 L agua, a partir de los últimos $30 \pm 2$ días de gestación hasta el destete. Los lechones descendientes del grupo T2 continuaron recibiendo el mismo aditivo que sus madres. La primera dosis aplicada a los cerditos fue un $\mathrm{mL}$ en mono dosis a los 10 días de edad. La dosificación en forma oral, fue variado: $1 \mathrm{~mL}$ en las semanas tres y cuatro; $1,5 \mathrm{~mL}$ en el quinto y sexto, $2 \mathrm{~mL}$ en el séptimo y octavo y $2,5 \mathrm{~mL}$ en las siguientes semanas hasta los 70 días de edad. Los animales del grupo control recibieron suero fisiológico en la misma cantidad que el grupo tratado. Los lechones a partir del destete (33 días de edad) recibieron el aditivo inoculado en $25 \mathrm{~g}$ de dieta basal y $50 \mathrm{~mL}$ agua.

Variables de respuesta. Para evaluar la repercusión del aditivo microbiano en las cerdas reproductoras y su descendencia; se determinaron como variables de respuesta los indicadores productivos (comportamiento de peso vivo en las diferentes etapas (cerdas madres) y peso al nacer de las crías), a los cerditos se pesaron a los 15 , 33, 52 y 70 días de edad. Para el mismo se utilizaron tres básculas romana URKO de 500, 50 y $20 \mathrm{~kg}$ de capacidad, respectivamente, previamente calibrada con un margen de error $\pm 0,25 \mathrm{~g}$. La prevalencia de diarreas y porcentajes de mortalidad fue valuado en todos los animales estudiados. Para la evaluación microbiana se realizó hisopado rectal a las cerdas madres y su descendencia. El muestreo se efectuó al destete (33 días de edad) y a los 15 días después de suspender el tratamiento (cerdas madres y lechones). El procedimiento experimental para la determinación de los indicadores se presenta a continuación.

Indicadores productivos. Con la información obtenida, se calculó la ganancia de peso (GP) por periodo al final del experimento.

Control de diarreas y mortalidad. Todos los animales en estudio fueron sometidos a un riguroso control clínico para detectar cambios de conducta, trastornos diarreicos y muertes. Con esta información se determinó la prevalencia de diarias y porcentaje de mortalidad. 
Procedimiento experimental para la toma de muestras y caracterización microbiana. Se seleccionaron al azar 30 lechones y todas las cerdas reproductoras de cada tratamiento y a estos se les practicó el hisopado rectal. Estas muestras se tomaron a las 7:00 am en hisopos con medios de transporte (DELTALAB ${ }^{\circ}$, España). Luego se trasladaron al laboratorio dentro de las primeras dos horas para su posterior procesamiento. La caracterización microbiana, consistió en la descripción de los organismos cultivados en placas Petri con medios selectivos agar MRS (Man, Rogosa y Sharpe M6411-500G, HEMEDIA®, India), Nutritivo (Nutr, 213000-BD Difco ${ }^{\mathrm{TM}}$, USA) y Agar Sabouraud (Sabrd 211584-BD BBL ${ }^{\mathrm{TM}}$, USA) de las muestras del hisopado rectal de las cerdas reproductoras y sus lechones. Estos, se incubaron por $24 \mathrm{~h}$ a $37^{\circ} \mathrm{C}$ y $30^{\circ} \mathrm{C}$ para bacterias y levaduras, respectivamente. Las placas con medio de cultivo MRS fueron cultivados en condiciones anaerobias utilizando la jarra GasPak Plus $^{\mathrm{TM}}$. Pasado este tiempo se desarrolló recuento celular de número de unidades formadoras de colonias (UFC), se cuantificó por conteo visual de las colonias, tinción de Gram, pruebas de catalasa y se observó al microscopio eléctrico (BA310 Binocular MOTIC, China). Para diferenciar a las características morfo-tintoriales, se utilizó la metodología descrita en Manual of Systematic Bacteriology Kandler y Weiss (1992). Todas las pruebas realizadas fueron por quintuplicado.
Análisis Estadístico. Los datos experimentales se procesaron con el paquete estadístico Statgraphic plus 15.1 para Windows. Se realizó el Análisis de Varianza según el diseño Completamente Aleatorizado y en los casos necesarios, se aplicó la Prueba de Comparación de Duncan (1955) para discriminar diferencias entre medias a $\mathrm{P}<0,05$.

\section{RESULTADOS Y DISCUSIÓN}

En la Tabla 2, se muestra el comportamiento de los indicadores productivos medidas durante el estudio. Al valorar el peso vivo de las cerdas reproductoras suplementadas con el preparado probiótico a partir de $30 \pm 2$ días antes de parto hasta el destete, las condiciones corporales fueron estables y la disminución de peso vivo fue menor a $5,2 \mathrm{~kg}$ en las cerdas madres del tratamiento $\mathrm{T} 2$ durante la lactación y hubo diferencia $(\mathrm{P}<0,05)$ con respecto a los animales del grupo control. El comportamiento productivo favorable de los lechones es reflejo de la respuesta del preparado probiótico adicionado a la dieta a partir del último mes de la gestación $\mathrm{y}$ esto hace que se logre mantener en los rangos normales de producción para esta especie y categorías (Cottney, et al., 2012; Ek-Mex, et al., 2015).

Tabla 2. Comportamiento productivo de las cerdas reproductoras y su descencia al suplementar aditivo microbiano en la dieta basal.

\begin{tabular}{lcccc}
\hline \multirow{2}{*}{\multicolumn{1}{c}{ Variables medidos }} & \multicolumn{2}{c}{ Tratamientos } & \multirow{2}{*}{ SEM } & p-valor \\
\cline { 2 - 3 } & T1 & T2 & & \\
\hline Peso vivo al inicial, kg (85 d de gestada) & 115,3 & 115,6 & 0,01 & 0,8511 \\
Peso vivo al Final, kg (al parto) & 103,1 & 108,4 & 0,12 & 0,0152 \\
Números de crías nacidas vivos /camada & 11 & 12 & - & - \\
Numero de crías nacidos muertos (U) & 7 & 0 & - & - \\
Peso de nacimiento de las crías (kg) & 1,12 & 1,40 & 0,06 & 0,0012 \\
\hline
\end{tabular}

Valores distintas en la misma fila difieren a $\mathrm{p}<0,05$ (Duncan, 1955). T1, dieta basal sin aditivo. T2, dieta más L. acidophilus más $S$ thermophilus y K. fragilis (L-4 UCLV). 
El aditivo probiótico suplementado a las cerdas madres en el último tercio de la gestación ayudó a mejorar significativamente $(\mathrm{P}<0,05)$ el comportamiento productivo sobre los animales que no consumieron. Así mismo, no hubo crías nacidas muertas en las camadas que consumieron probiótico. Similares resultados fueron reportados por Cottney et al. (2012) al suplementar un cultivo mixto de $L$ acidophilus y L rhamnosus a cerdas reproductoras. El peso de las crías al nacer provenientes de las cerdas madres tratadas fue superior a $35 \mathrm{~g}$ sobre los descendientes que no consumieron probiótico. Los resultados del presente estudio, coinciden en parte con los reportados por Ayala et al. (2014), quienes atribuyen a los probióticos las mejorías sobre el proceso de la digestión y absorción de los nutrientes, unido al anabolismo gestacional en la cerda, que a su vez permite realizar un mejor aprovechamiento de sus dietas favoreciendo al crecimiento del feto en la etapa final de la gestación (Gutiérrez et al., 2014).

En la Tabla 3, se puede apreciar el comportamiento del promedio de ganancia de peso en las diferentes etapas productivas del cerdo. En la etapa neonato y cría, los lechones del grupo T2 obtuvieron en peso superior a $1,5 \mathrm{~kg}$ sobre los animales del grupo control, mientras que, en la etapa pre-ceba la ganancia fue mayor a $2 \mathrm{~kg}$, sobre los animales que no consumieron el preparado probiótico. En ambas casos hubo diferencias $(\mathrm{P}<0,05)$ con respecto a los animales del grupo control. El periodo preceba fue el de mejor $(\mathrm{P}<0,05)$ comportamiento con respecto a otras etapas productivas.

La repercusión positiva sobre los parámetros productivos en los lechones, al incluir de los microorganismos probióticos en la dieta, fue posiblemente, debido a la regeneración de las microvellosidades, mayor absorción y mejor aprovechamiento de nutrimientos, lo que se pudo reflejar de manera positiva en el comportamiento bioproductivo (Chaytor et al., 2011).

García et al. (2012) reportaron mejorar la asimilación de nutrientes, en efecto aumenta la ganancia de peso, cuando los cerdos consumieron microorganismos probióticos en la dieta. Por su parte, Ihara et al. (2013) informaron mejorar la digestibilidad, la producción de vitaminas del complejo B y cofactores de crecimiento en cerdos. efectos positivos sobre el comportamiento productivo y la salud de los lechones al suplementar probiótico en la dieta (Londoño et al., 2016).

En la Figura 1, se observa la prevalencia de diarreas en las diferentes etapas productivas de los cerdos.

Tabla 3. Comportamiento de peso vivo en las diferentes etapas del cerdo.

\begin{tabular}{|c|c|c|c|c|}
\hline \multirow{2}{*}{$\begin{array}{c}\text { Etapas } \\
\text { Productivas }\end{array}$} & \multicolumn{2}{|c|}{ Tratamientos } & \multirow{2}{*}{ SEM } & \multirow{2}{*}{$p$-valor } \\
\hline & T1 & $\mathrm{T} 2$ & & \\
\hline Neonato & 4,75 & 6,25 & 0,11 & $<, 0001$ \\
\hline Crías & 4,50 & 6,15 & 0,13 & $<, 0001$ \\
\hline Crecimiento & 4,45 & 5,45 & 0,13 & $<, 0001$ \\
\hline Pre-ceba & 7,53 & 9,53 & 0,03 & $<, 0001$ \\
\hline
\end{tabular}

Valores distintas en la misma fila difieren a p<0,05 (Duncan 1955). T1, dieta basal sin aditivo. $\mathrm{T} 2$, dieta más L. acidophilus más $S$ thermophilus y K. fragilis (L-4 UCLV). 


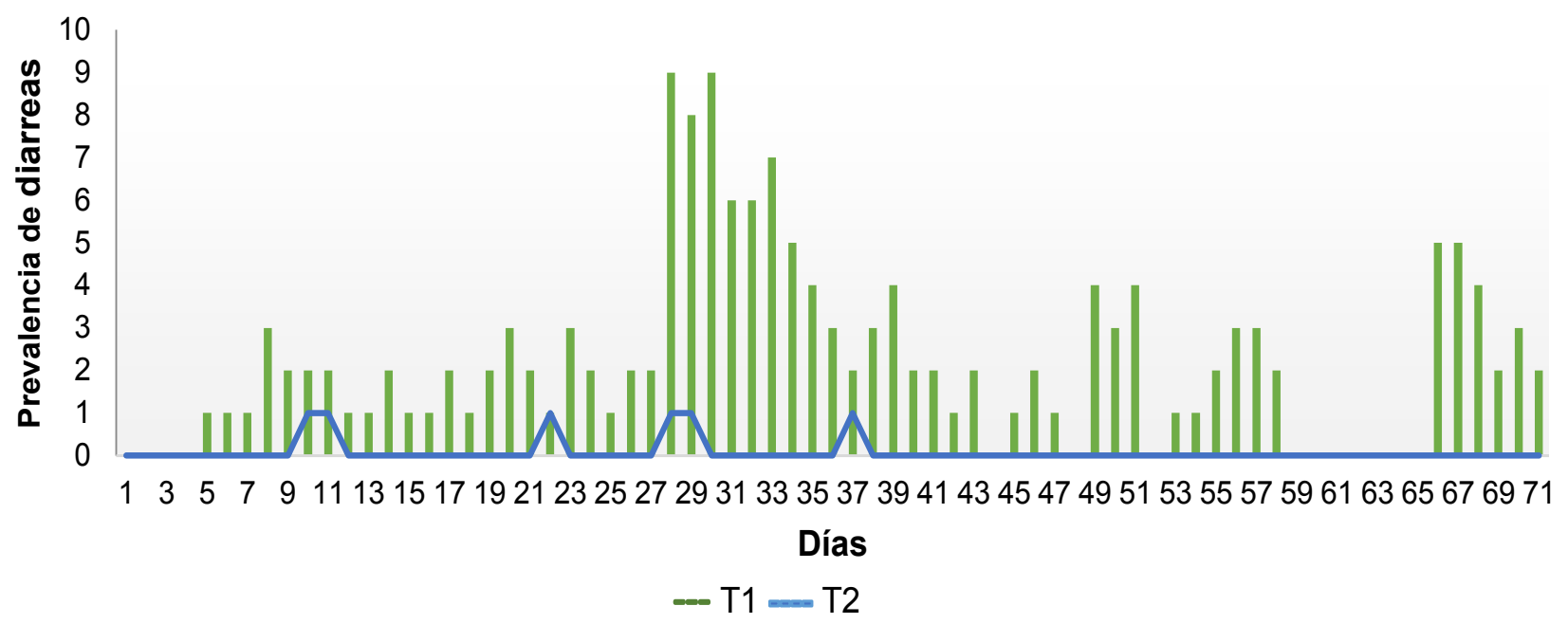

T1, dieta basal sin aditivo. T2, dieta más L. acidophilus más $S$. thermophilus y K. fragilis (L-4 UCLV).

Figura 1. Prevalencia de los trastornos diarreicos de lechones en el experimento.

La presencia de las diarreas fue mayor $(\mathrm{P}>0,05)$ en el grupo T1, con respecto a los animales del tratamiento T2. La disminución diarrea en los animales que consumieron el preparado probiótico (Figura 1) en los primeros días posteriores al destete, momento crítico en la vida del animal. Fue posiblemente por la acción de los microorganismos probióticos introducidos con la dieta debido a su capacidad de formar metabolitos segundarios como son las bacteriocinas, ácido láctico, peróxido, entre otros. En este sentido, Ayala et al. (2014) observaron disminuir el porcentaje de diarreas y muertes al incluir bacterias ácido lácticas en la dieta de los lechones.

Zhao et al. (2015) redujeron $(\mathrm{P} \leq 0,05)$ la prevalencia de diarreas y muertes al suplementar un cultivo mixto de bacterias acido lácticas en cerdos destetados. Similares resultados fueron reportados por Vélez-Zea et al. (2015) con la suplementación de Bacillus subtilis y Bacillus licheniformis. Por su parte, Rajput et al. (2013) y Lähteinen et al. (2015) además de mejorar la salud, observaron aumentar el comportamiento productivo en cerdos que consumieron los probióticos con respecto al grupo control. Los resultados obtenidos en el presente estudio, corroboran las bondades que prestan los preparados probióticos sobre la salud de los cerdos en las diferentes etapas productivas.

En la Tabla 4, se aprecia las características morfotintoriales de los microorganismos aislados a partir del hisopado rectal de las cerdas madres que consumieron los preparados microbianos, en la evaluación realizada al destete y a los 15 días posteriores a la suspensión del probiótico.

Las colonias uniformares fue mayor $(\mathrm{P}<0,05)$ en las muestras provenientes de los animales que consumieron el preparado probiótico. Así mismo, hubo mayor $(\mathrm{P}<0,05)$ número de células unidades formadoras de colonias (UFC) en el mismo grupo. $\mathrm{Al}$ observar, en el microscopio eléctrico los microrganismos con formas de bacilos cortos, largos y en cadena, cocos aislados, pares y en cadenas, Gram positivo y catalasa negativo fue mayor $(\mathrm{P}<0,05)$ en el T2 con respecto al grupo control.

Al evaluar las muestras que fueron cultivadas en placas con agar Sabouraud las colonias mostraron un color amarrillo cremoso con cierto abultamiento, asimismo, hubo diferencia $(\mathrm{P}<0,05)$ con respecto al grupo control. 
Tabla 4. Nivel de crecimiento celular y la característica morfo-tintorial de las colonias del hisopado rectal de las cerdas reproductoras (híbridos CC21) suplementadas con probiótico.

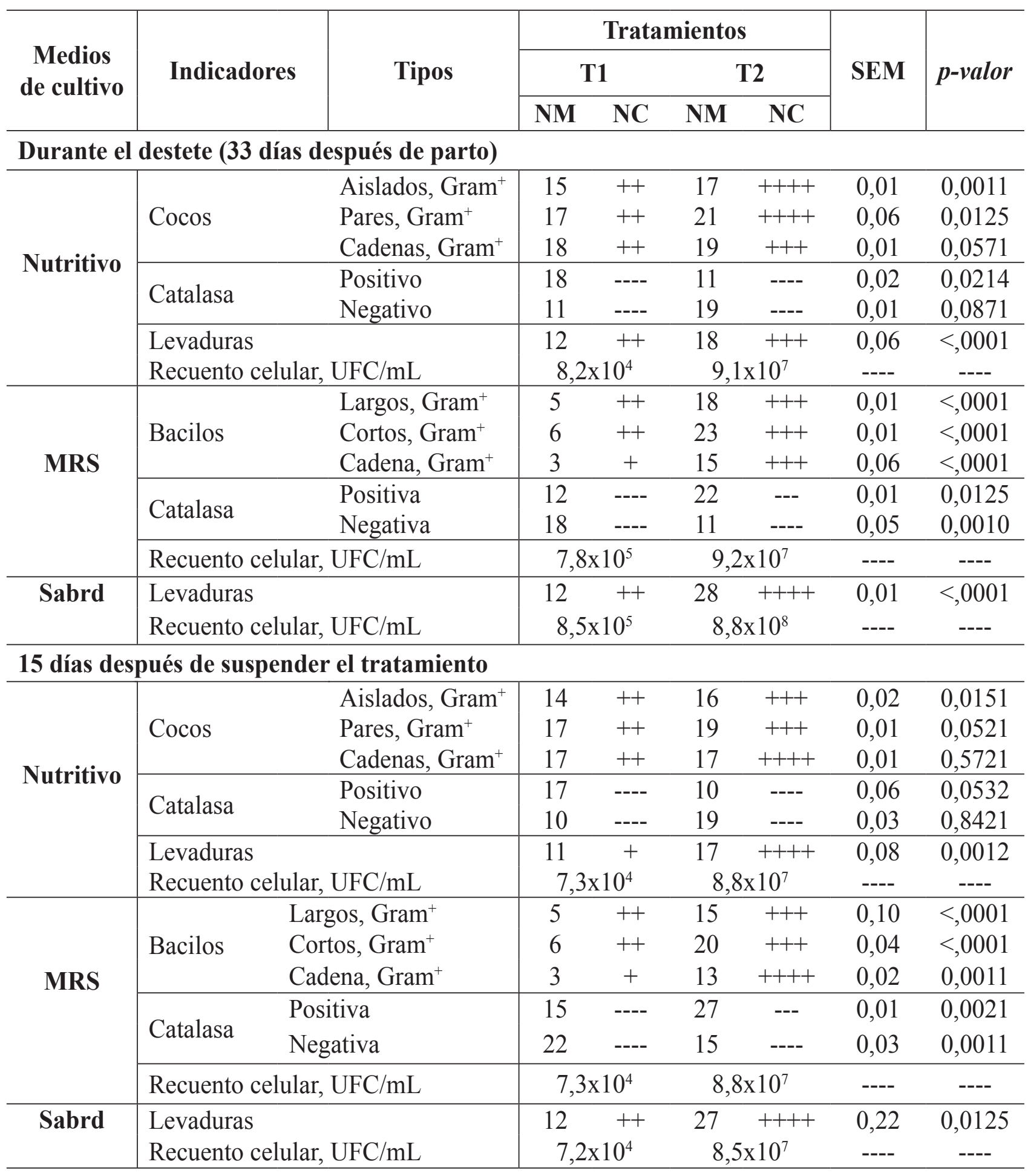

Valores distintas en la misma fila difieren a p <0,05 (Duncan, 1955). T1, dieta basal sin aditivo. T2, dieta más L. acidophilus más $S$ thermophilus y K. fragilis (L-4 UCLV). NM, número de muestras. NC, nivel de crecimiento. -, no crecimiento. +, Poco crecimiento. ++, Regular crecimiento. +++, Mayor crecimiento. ++++, Excelente crecimiento. MRS, Man, Rogosa y Sharpe Sabrd, Agar Sabouraud. UFC, unidades formadoras de colonias. Caracterización microbiana según la metodología descrita por Kandler y Weiss (1992). 
Del mismo modo, en el muestreo realizado 15 días después de suspender el tratamiento, las placas Petri con muestras procedentes de las cerdas madres que consumieron preparado probiótico, mantenían valores similares a los obtenidos en el destete en todos los indicadores evaluados, pero al comparar entre grupos hubo diferencias $(\mathrm{P}<0,05)$ en todas las características morfológicas de los microorganismos en estudio (Tabla 4).

Los resultados obtenidos en el presente estudio coinciden en parte con los reportados por Rajput et al. (2013), quienes reportaron algunas características morfológicamente similares a los observados en el estudio, entre los que se destacan microorganismos de géneros Lactobacillus y Bifidobacterium, dichas bacterias mayoritariamente son de origen intestinal y utilizadas de forma tradicional en fermentaciones alimenticias de uso veterinario.

Por su parte, Sun et al. (2015) observaron, al introducir microorganismos vivos como aditivos en la dieta para los animales, la modificación de la composición de la microbiota intestinal y su efecto potencial sobre la absorción de nutrientes y minerales en el huésped (Vélez-Zea et al., 2015). Unido a esto, Zhao et al. (2015) manifiestan, que los microorganismos vivos introducidos buscan un nicho adecuado donde compiten e interaccionan entre sí, constituyendo finalmente una población relativamente estable y compleja en la microbiota natural del hospedero, no obstante, esta estabilidad puede ser alterada por cambios dietéticos 0 ambientales que son sometidos los animales (Londoño et al., 2016). Sin embargo, es limitada la información sobre las funciones que desempeñan en el metabolismo de nutrientes y el tiempo de permanencia en el tracto gastrointestinal de los animales (Patil et al., 2015).
En la Tabla 5, se aprecia el crecimiento microbiano en diferentes medios selectivos y descripción del tipo de microorganismo en el muestreo realizado a lechones durante el destete y 15 días después de suspender tratamiento. Las colonias uniformares fueron mayores $(\mathrm{P}<0,05)$ en las muestras provenientes de los lechones del grupo T2. Así mismo, hubo mayor $(\mathrm{P}<0,05)$ número de células unidades formadoras de colonias (UFC) en el T2 con respecto a T1. Igualmente, los organismos en formas de bacilos cortos, largos y en cadenas, cocos aislados, pares y en cadenas, Gram positivo y catalasa negativa fueron mayores $(\mathrm{P}<0,05)$ en el $\mathrm{T} 2$. También hubo mayor $(\mathrm{P}<0,05)$ incremento de colonias de color amarrillo cremoso con cierto abultamiento en placas con agar Sabouraud en el T2 con respecto al grupo control. En todos los medios selectivos, las muestras provenientes del grupo T2 mostraron mayor $(\mathrm{P}<0,05)$ crecimiento microbiano con respecto al grupo control.

En cuanto a la evaluación realizada 15 días después de suspender el tratamiento, mantenían características similares a los obtenidos en la evaluación realizada al destete, pero al comparar entre grupos hubo diferencia $(\mathrm{P}<0,05)$ en algunos indicadores medidos. Los resultados reportados en el presente estudio indican que los microorganismos probióticos incluidos en la dieta de los cerdos pudieron adherirse a los diferentes segmentos del tracto gastrointestinal, esto demuestra que la microbiota natural podría estar modificada por los organismos benéficos (Patil et al., 2015). 
Tabla 5. Nivel de crecimiento celular y las características morfo-tintoriales de las colonias del hisopado rectal de los lechones tratados con probiótico.

\begin{tabular}{|c|c|c|c|c|c|c|c|c|}
\hline \multirow{3}{*}{$\begin{array}{l}\text { Medios de } \\
\text { cultivo }\end{array}$} & \multirow{3}{*}{ Indicadores } & \multirow{3}{*}{ Tipos } & \multicolumn{4}{|c|}{ Tratamientos } & \multirow{3}{*}{ SEM } & \multirow{3}{*}{ p-valor } \\
\hline & & & \multicolumn{2}{|c|}{ T1 } & \multicolumn{2}{|c|}{$\mathbf{T 2}$} & & \\
\hline & & & NM & $\mathbf{N C}$ & NM & $\mathbf{N C}$ & & \\
\hline \multicolumn{9}{|c|}{ Durante el destete (33 días después de parto) } \\
\hline \multirow{7}{*}{ Nutritivo } & \multirow{3}{*}{ Cocos } & Aislados, Gram ${ }^{+}$ & 18 & +++ & 38 & ++++ & 0,03 & 0,0214 \\
\hline & & Pares, Gram ${ }^{+}$ & 24 & ++++ & 38 & ++++ & 0,01 & 0,0011 \\
\hline & & Cadenas, Gram ${ }^{+}$ & 22 & +++ & 31 & ++++ & 0,06 & 0,0125 \\
\hline & \multirow{2}{*}{ Catalasa } & Positivo & 28 & ---- & 15 & ---- & 0,02 & 0,0012 \\
\hline & & Negativo & 15 & ---- & 38 & ---- & 0,03 & 0,0125 \\
\hline & \multicolumn{2}{|l|}{ Levaduras } & 25 & ++ & 40 & +++ & 0,06 & $<, 0001$ \\
\hline & \multicolumn{2}{|c|}{ Recuento celular, UFC/mL } & \multicolumn{2}{|c|}{$8,5 \times 10^{6}$} & \multicolumn{2}{|c|}{$9,3 \times 10^{8}$} & ---- & ---- \\
\hline \multirow{6}{*}{ MRS } & \multirow{3}{*}{ Bacilos } & Largos, Gram ${ }^{+}$ & 25 & + & 59 & ++++ & 0,01 & $<, 0001$ \\
\hline & & Cortos, Gram ${ }^{+}$ & 27 & ++++ & 56 & ++++ & 0,01 & $<, 0001$ \\
\hline & & Cadenas, Gram ${ }^{+}$ & 30 & ++ & 52 & ++++ & 0,06 & $<, 0001$ \\
\hline & \multirow{2}{*}{ Catalasa } & Positiva & 15 & $\begin{array}{ll}-- \\
\end{array}$ & 34 & --- & 0,01 & 0,0125 \\
\hline & & Negativa & 24 & --- & 10 & --- & 0,03 & 0,0021 \\
\hline & \multicolumn{2}{|c|}{ Recuento celular, UFC/mL } & \multicolumn{2}{|c|}{$8,1 \times 10^{5}$} & \multicolumn{2}{|c|}{$9,1 \times 10^{8}$} & ---- & ---- \\
\hline \multirow[t]{2}{*}{ Sabrd } & \multicolumn{2}{|l|}{ Levaduras } & 25 & ++ & 63 & ++++ & 0,01 & $<, 0001$ \\
\hline & \multicolumn{2}{|c|}{ Recuento celular, UFC/mL } & \multicolumn{2}{|c|}{$8,3 \times 10^{5}$} & \multicolumn{2}{|c|}{$9,4 \times 10^{7}$} & ---- & ---- \\
\hline
\end{tabular}

15 días después de suspender el tratamiento

\begin{tabular}{|c|c|c|c|c|c|c|c|c|}
\hline \multirow{5}{*}{ Nutritivo } & Cocos & $\begin{array}{l}\text { Aislados, Gram } \\
\text { Pares, Gram } \\
\text { Cadenas, Gram } \\
\text { + }\end{array}$ & $\begin{array}{l}12 \\
18 \\
20 \\
\end{array}$ & $\begin{array}{c}++ \\
++++ \\
+++ \\
\end{array}$ & $\begin{array}{l}28 \\
38 \\
33 \\
\end{array}$ & $\begin{array}{l}++++ \\
++++ \\
++++\end{array}$ & $\begin{array}{l}0,02 \\
0,01 \\
0,21 \\
\end{array}$ & $\begin{array}{l}<, 0001 \\
<, 0001 \\
0,0012 \\
\end{array}$ \\
\hline & \multirow{2}{*}{ Catalasa } & Positiva & 24 & $\begin{array}{ll}--- \\
--\end{array}$ & 12 & ---- & 0,10 & $<, 0001$ \\
\hline & & Negativa & 28 & ---- & 13 & ---- & 0,10 & 0,0021 \\
\hline & \multicolumn{2}{|c|}{ Levaduras } & 25 & + & 40 & +++ & 0,15 & $<, 0001$ \\
\hline & \multicolumn{2}{|c|}{ Recuento celular, UFC/mL } & \multicolumn{2}{|c|}{$8,2 \times 10^{4}$} & \multicolumn{2}{|c|}{$9,3 \times 10^{8}$} & ---- & ---- \\
\hline \multirow{6}{*}{ MRS } & \multirow{3}{*}{ Bacilos } & Largos, Gram ${ }^{+}$ & 25 & ++++ & 59 & ++++ & 0,01 & $<, 0001$ \\
\hline & & Cortos, Gram ${ }^{+}$ & 27 & ++ & 56 & ++++ & 0,02 & $<, 0001$ \\
\hline & & Cadenas, Gram ${ }^{+}$ & 30 & ++ & 52 & ++++ & 0,10 & 0,0012 \\
\hline & \multirow{2}{*}{ Catalasa } & Positiva & 18 & ---- & 42 & --- & 0,02 & 0,0021 \\
\hline & & Negativa & 25 & ---- & 14 & ---- & 0,01 & 0,0011 \\
\hline & \multicolumn{2}{|c|}{ Recuento celular, UFC/mL } & \multicolumn{2}{|c|}{$7,2 \times 10^{5}$} & \multicolumn{2}{|c|}{$9,2 \times 10^{8}$} & $\begin{array}{ll}---- \\
\end{array}$ & ---- \\
\hline \multirow[t]{2}{*}{ Sabrd } & \multicolumn{2}{|c|}{ Levaduras } & 25 & ++ & 63 & ++++ & 0,05 & $<, 0001$ \\
\hline & \multicolumn{2}{|c|}{ Recuento celular, UFC/mL } & \multicolumn{2}{|c|}{$7,7 \times 10^{4}$} & \multicolumn{2}{|c|}{$9,1 \times 10^{7}$} & ---- & ---- \\
\hline
\end{tabular}

Valores distintos en la misma fila difieren a $\mathrm{p}<0,05$ (Duncan 1955). T1, dieta basal sin aditivo. T2, dieta más $L$. acidophilus más $S$ thermophilus y $K$. fragilis (L-4 UCLV). NM, número de muestras. NC, nivel de crecimiento. -, no crecimiento. +, Poco crecimiento. ++, Regular crecimiento. +++, Mayor crecimiento. ++++, Excelente crecimiento. MRS, Man, Rogosa y Sharpe. Sabrd, Agar Sabouraud. Caracterización microbiana según la metodología descrita por Kandler (1992). 
Es importante suplementar en cantidad relativamente alta de UFC introduciendo en la dieta, para lograr una mayor implantación y permanencia en el tracto gastrointestinal y que estos promuevan la estimulación del sistema inmune, evitando la colonización de los agentes patógenos en el hospedero (Gutiérrez et al., 2014). De manera regular, en la literatura se informa que cuando se incluyen microorganismos vivos como aditivos en las dietas, estos influyen en la salud disminuyendo la carga microbiana de los agentes patógenos en el huésped (Sun et al., 2015; Gutiérrez et al., 2014).

Las caracterizadas morfológicamente de los microorganismos del presente estudio se asemejan con las especies pertenecientes al género $L$ acidophilus, por Patil et al. (2015), streptococcus aisladas de intestino del cerdos (Castro y Martínez, 2015) y levaduras del genero Kluyveromyces reportado por Miranda et al. (2017). Por su parte, González et al. (2016) observaron que los microorganismos introducidos por vía oral, se asocian en la mucosa intestinal para cumplir con las funciones probióticas. Los resultados obtenidos corroboran la presencia de los microorganismos en el tracto gastrointestinal. En otros estudios, se ha demostrado la variación microbiana en el tubo digestivo de los cerdos que consumieron aditivos probióticos (Patil et al., 2015). Por lo tanto, el uso de los probióticos es una buena alternativa para mantener un equilibrio en la microbiota natural en lechones neonatos. Así mismo, la salud de los animales se ve influenciada por las condiciones ambientales y de manejo durante el destete, donde sufre cambios drásticos en su régimen social y alimentación, que desencadenan en una serie de cambios morfológicos (a nivel epitelial) y fisiológicos, que lo hacen susceptible a enfermedades de tipo bacteriano (Yin et al., 2014; Lähteinen et al., 2015).

Los exámenes microbiológicos como el hisopado rectal en los lechones, ayudan a evaluar el nivel de microorganismos presentes a este nivel (Londoño et al., 2016). La cantidad de muestras crecidas en medios específicos a partir de hisopado rectal de las muestras provenientes de los lechones que consumieron probiótico hasta 70 días de edad, fue superior a los reportados por Cottney et al. (2012). Los organismos con capacidad probiótica se caracterizan por su capacidad de resistir a condiciones ambientales del tracto gastrointestinal, a cambios de $\mathrm{pH}$ y su estructura morfológica puede ser en forma de cocos, bacilos y Gram positivos no esporulados. Normalmente, un gran número de éstos microorganismos son aislados desde el suelo, heces y tracto gastrointestinal del animal (Castro y Martínez, 2015).

\section{CONCLUSIONES}

Las cerdas reproductoras que consumieron probiótico en el último tercio de la gestación obtuvieron mayor peso al parto, asimismo, sus descendientes nacieron con mayor peso. Además, los indicadores productivos de los lechones fueron mejores en todas las etapas evaluadas, se logro reducir los trastornos diarreicos y las muertes. Así mismo, hubo mayor presencia de organismos en forma de cocos (aislados, pares y en cadena), bacillos (cortos largos y cadena), Gram positivo, catalasa negativo y levaduras de color amarrillo cremoso con cierto abultamiento, en las muestras del hisopado rectal cultivados en medios selectivos.

Conflicto de intereses: Los autores declaran que no hay conflicto de interés.

\section{REFERENCIAS BIBLIOGRÁFICAS}

Ayala, L., Bocourt, R., Castro, M., Dihigo, L. E., Milián, G., Herrera, M., \& Ly, J. (2014). Desarrollo de órganos digestivos en cerditos descendientes de madres que consumieron un probiótico, antes del parto y durante la lactancia. Revista Cubana de Ciencia Agrícola. 48(2): 133-136. 
Castro, M., Martínez, M. (2015). La alimentación porcina con productos no tradicionales. Revista Cubana de Ciencia Agrícola. 49(2): 189-196

Chaytor, A.C., Hansen, J.A., Heugten, E.V., Todd-See, T. \& Woo-Kim, K. (2011). Occurrence and Decontamination of Mycotoxins in Swine Feed. Asian-Australasian Journal of Animal Sciences. 24(5): 723-738. doi: https://doi.org/10.5713/ ajas.2011.10358

Coates, J., Corns, P.J., Juarez, A., MacDonald, R., McCulley, N., Melody, B., Minton, A., Molinari, R., Montes De Oca, H., Mosqueira, P., Neill, C., Pinilla, J.C., Piva, J. \& Teuber, R. (2013). Manual PIC de Manejo de Hembras y Primerizas. Hendersonville: PIC North America.

Cottney, P.D., Magowan, E., Ball, E. \& Gordon A. (2012). Effect of oestrus number of nulliparous sows at first service on first litter and lifetime performance. Livestock Science. 146(1): 5-12. doi: http://dx.doi.org/10.1016/j.livsci.2012.02.013

Duncan, D.B. (1955). Multiple range and multiple F test. Biometrics. 11(1): 1-42.

Ek-Mex, J.E., Segura-Correa, J.C., Alzina-López, A. \& Aké-López R. (2015). Factores ambientales que afectan algunas características postdestete de las cerdas en el trópico de México. Archivo Medicina Veterinaria. 47(1): 45-51. doi: 10.4067/ S0301-732X2015000100009

García, A.C., De Loera, Y.G., Yagüe, A.P., Guevara, J.A. \& García, C. (2012). Alimentación práctica del cerdo. Revista Complutense de Ciencias Veterinarias. 6(1): 21-47. doi: http://dx.doi. org/10.5209/rev_RCCV.2012.v6.n1.38718

González, M., Figueroaa, J.L., Vaquerab, H., SánchezTorresa, M.T., Ortegaa, M.E., Copadoc, J.M.F. \& Martínez, J.A. (2016). Meta análisis del efecto de dietas bajas en proteína y adicionada con aminoácidos sintéticos para cerdos machos castrados en finalización. Archivos de medicina veterinaria. 48(1): 51-59. doi: http://dx.doi. org/10.4067/S0301-732X2016000100007

Gutiérrez, L.A., Bedoya, 0. \& Ríos M. (2014). Evaluación de parámetros productivos en cerdos (Sus scrofa domesticus) suplementados con microorganismos probióticos nativos. Journal of Agriculture and Animal Sciences. 3(2): 48-55.

Ihara, Y., Hyodo, H., Sukegawa, S., Murakami, H. \& Morimatsu, F. (2013) Isolation, characterization, and effect of administration in vivo, a novel probiotic strain from pig feces. Animal Science Journal. 84(5): 434-441. doi: 10.1111/ asj.12020.

Kandler, 0. \& Weiss, N. (1992). Regular nonsporing Gram-positive rods. pp. 1208-1260. En: P. H. A. Sneath, M. S. Mair, M. E. Sharpe y J. G. Holt. Bergey's Manual of Systematic Bacteriology, 10th edition, vol. 2. Baltimore: The Williams and Wilkins Co.

Lähteinen, T., Rinttilä, T., Koort, M.K., Kant. R., Levonen, K., Jakava-Viljanen, M., Björkroth, J. \& Palva, A. (2015). Effect of a multi species lactobacillus formulation as a feeding supplement on the performance and immune function of piglets. Livestock Science. 180(1): 164-171. doi: http:// dx.doi.org/10.1016/j.livsci.2015.07.016

Londoño, A., Lallès, J.P. \& Parra, J. (2016). Effect of probiotic strain addition on digestive organ growth and nutrient digestibility in growing pigs. Revista Facultad Nacional de Agronomía. 69(2): 7911-7918. doi: 10.15446/rfna. v69n2.59136.

Miranda, J.E., Marin, A. \& Baño, D. (2017). Elaboration of a bioprepared with probiotic effect from a mixed culture of lactic bacteria and yeasts. Revista Bionatura. 2(1): 273-275. doi: 10.21931/ $\mathrm{RB} / 2017.02 .01 .6$ 
NRC - National Research Council. (1998). The nutrient requirements of beef cattle. 10th ed. Washington, DC, USA: National Academy Press.

Patil, A.K., Kumar. S., Verma, A.K. \& Baghel, P.S. (2015). Probiotics as Feed Additives in Weaned Pigs. Livestock Research International. 3(2): 31-39.

Rajput, I. R., Li, L.Y., Xin, X., Wu, B.B., Juan, Z.L., Cui, Z.W. \& Li, W.F. (2013). Effect of Saccharomyces boulardii and Bacillus subtilis B10 on intestinal ultrastructure modulation and mucosal immunity development mechanism in broiler chickens. Poultry science. 92(4): 956-965. doi: 10.3382/ps.2012-02845.

Sun, Y., Park, I., Guo, J., Weaver, A. \& Woo, S. (2015). Impacts of low level aflatoxin in feed and the use of modified yeast cell wall extract on growth and health of nursery pigs. Journal Animal Nutrition. 1(3): 177-183. doi: 10.1016/j. aninu.2015.08.012

Vélez-Zea, J.M., Gutiérrez-Ramírez, L.A. \& Montoya-Campuzano, O.I. (2015). Bactericidal Evaluation of Lactic Acid Bacteria Isolated in Sow Colostrum Against Salmonella tiphymurium. Revista Facultad Nacional de Agronomía, Medellín. 68(1): 7481-7486. doi: https://dx.doi. org/10.15446/rfnam.v68n1.47834

Yin, J., Ren, W., Duan, J., Wu, L., Chen, S., Li, T., Yin, Y. $\&$ Wu, G. (2014). Dietary arginine supplementation enhances intestinal expression of SLC7A7 and SLC7A1 and ameliorates growth depression in mycotoxin-challenged pigs. Amino Acids. 46(4): 883-892. doi: 10.1007/s00726-0131643-5

Zhao, P.Y., Jung, J.H. \& Kim, I.H. (2015). Effect of mannan oligosaccharides and fructan on growth performance, nutrient digestibility, blood profile, and diarrhea score in weanling pigs. Journal Animal of Science. 90(3): 33-839. doi: 10.2527/jas.2011-3921. 